Jpn. J. Genet. (1987) 62, pp. 21-25

\title{
Interspecific conjugation between distantly related species of Paramecium
}

\author{
By Hiroshi ENDOH ${ }^{1)}$ \\ Biological Institute, Tohoku University, Sendai 980
}

(Received November 20, 1986)

\begin{abstract}
Interspecific conjugation was induced artificially between the distantly related species belonging to the two different morphological groups, 'aurelia' and 'bursaria' groups, of Paramecium. Cytological observations showed that the interspecific conjugants underwent normal nuclear changes, including meiosis. However, nuclear changes of co-conjugant cells did not synchronize each other. These results suggest that conjugation in Paramecium involves non-species-specific interactions and its interactions are sufficient for nuclear activation.
\end{abstract}

\section{INTRODUCTION}

Conjugation in ciliated protozoan Paramecium is a sexual process that is comparable to fertilization in metazoan. It involves a series of developmental events, such as meiosis, fertilization and nuclear differentiation (Metz 1954; Sonneborn 1957; Hiwatashi 1981, 1985). The first step of conjugation is sexual cell agglutination called mating reaction which is a specific cell interaction by the cilia of complementary mating types (Sonneborn 1937). In the second step, cells adhere at the anterior cell surfaces of cell bodies where cilia degenerate (Watanabe 1978). Following this cell union, a third connection including cytoplasmic exchange occurs from anterior to posterior end of the cell.

Paramecium species are classified into two groups, 'aurelia' and 'bursaria' groups based on their morphology (Wichterman 1986), the specificity of the immaturity substances which control the sexual immaturity after conjugation (Miwa 1979) and isozyme pattern of hemoglobin (Usuki and Irie 1982a, 1982b, personal communication). Hiwatashi (1951), Miyake (1968) and Tsukii and Hiwatashi (1983) demonstrated that the process following mating reaction does not involve mating-type specificity or species (including syngen and species complex) specificity, although the observations had been restricted to Paramecium belonging to 'aurelia' group. In contrast, it had been believed that there was the self-incompatibility that excludes the homotypic pair formation in P. bursaria. However, selfing pairs consisting of the same

1) Present address: Institute of Biological Sciences, University of Tsukuba, Ibaraki 305, Japan. 
mating type do appear when conjugation is induced by mixing the detached cilia of complementary mating type (Endoh et al. 1987) or by the treatment of conjugation inducing-chemicals in chemically inducible mutants (Endoh, unpublished). This fact promotes me to test the possibility that the two distantly related groups of Paramecium have the potentials that exceed the prezygotic barrier of species. In order to test this possibility, experiments were designed to induce artificially interspecific conjugation between the two groups of Paramecium.

\section{MATERIALS AND METHODS}

Stock $\mathrm{hr}^{\mathrm{d}}$ (high reacter, mating type VII) in $P$. tetraurelia and stock $27 \mathrm{aG} 3$ (mating type V) in $P$. caudatum from the 'aurelia' group and stock T151 (mating type IV) in P. bursaria from the 'bursaria' group were used in this study. $\mathrm{hr}^{\mathrm{d}}$ is a mutant whose mating reaction lasts long and natural autogamy does not occur (Sonneborn 1974). 27aG3 (Tsukii and Hiwatashi 1979) is an exautogamous clone, derived from an early mature mutant (Myohara and Hiwatashi 1979). T151 is a mutant in which conjugation can be induced chemically (Endoh, unpublished). Cells were grown in Ca-poor fresh lettuce juice medium inoculated with Klebsiella pneumoniae one day before use (Hiwatashi 1968, Miyake 1968). Conjugation in Paramecium can be induced not only by the interaction of complementary mating types but also by certain chemical agents (Miyake 1958, 1968). In all three species, the chemical induction of conjugation was successful in the condition of $10 \mu \mathrm{M} \mathrm{Ca}{ }^{2+}, 8 \mathrm{mM}$ $\mathrm{K}^{+}, 40 \mu \mathrm{g} / \mathrm{ml}$ acriflavine, and $100 \mathrm{mM}$ acetamide. Conjugating pairs began to appear $40 \mathrm{~min}$ in P. tetraurelia, $60 \mathrm{~min}$ in $P$. caudatum and $2 \mathrm{hrs}$ in P.bursaria, respectively after treatment of chemical agents. In order to induce pairs simultaneously among three species, chemical inductions were started at different times. Cells of each two species were mixed just before intraspecific conjugants appeared.

\section{RESULTS AND DISCUSSION}

When conjugation was induced chemically between $P$. bursaria and $P$. caudatum, interspecific conjugants were observed besides each intraspecific ones (Figure 1A and C). At 5 hrs after the induction of conjugation, micronucleus in the co-conjugant cell of $P$. caudatum was in the crescent stage that is equivalent to pachytene-diplotene at meiotic prophase (Sugai 1974, 1976). In contrast, at $6 \mathrm{hrs}$ after the induction, micronucleus in P. bursaria also entered the premeiotic $\mathrm{S}$ phase that is identified as a swollen micronucleus. Thus, nuclear changes proceeded depending upon the original time schedule of conjugation of each species and did not synchronize each other. Interspecific conjugants between $P$. bursaria and $P$. tetraurelia are shown in 

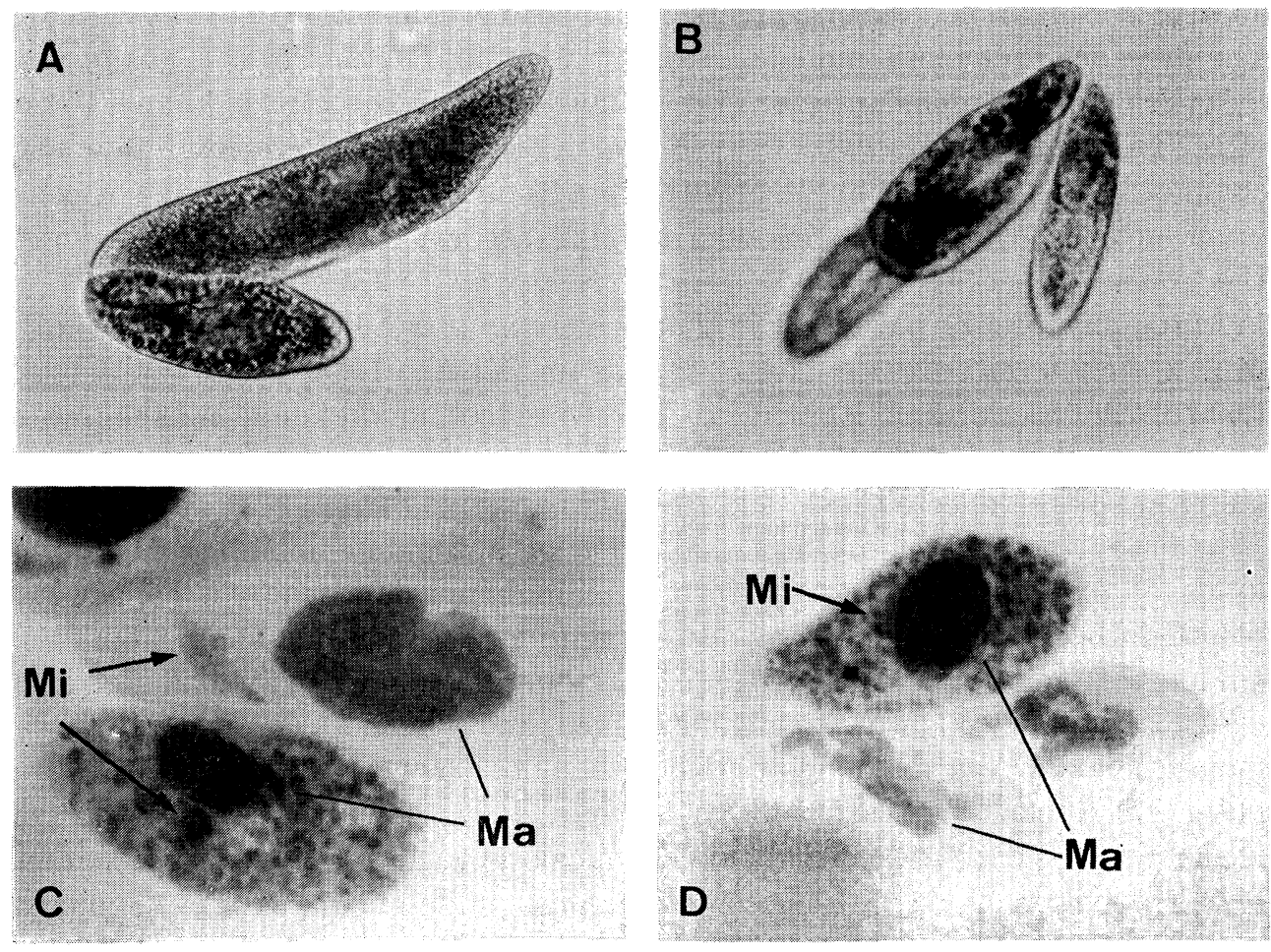

Fig. 1. A: Photomicrographs of interspecific conjugation between P. bursaria (lower) and $P$. caudatum (upper). B: Interspecific conjugation between $P$. bursaria (left) and $P$. tetraurelia (right). C: Micronucleus entering the crescent shaped stage equivalent to pachytene-diplotene at meiotic prophase in $P$. caudatum (upper) and swollen stage (premeiotic S phase) in P. bursaria (lower). 5-6 hrs after induction of conjugation. D: Macronucleus in P. tetraurelia (lower) entering the stage of skein formation specific for the sexual process and micronucleus in $P$. bursaria (upper) entering the swollen stage. 4-6 hrs after induction of conjugation. Symbiotic algae, zoochlorella, are seen in cells of $P$. bursaria. Mi: Micronucleus Ma: Macronucleus

Figure 1B and D. In P. tetraurelia, skein formation specific for the sexual process began $4 \mathrm{hrs}$ after the beginning of induction of conjugation. On the other hand, micronucleus of co-conjugant cells of $P$. bursaria entered the premeiotic $S$ phase $6 \mathrm{hrs}$ after the beginning. Nuclear changes also did not proceed synchronously in both partner cells of conjugants. The possible explanations to this are the followings: when conjugation is induced, there should be the mechanism that synchronizes the process of nuclear changes by the exchange of cytoplasm through the cell connection. In the interspecific conjugation, such mechanism may not be effective. A difference of the specificity of immaturity substances exists between 'aurelia' and 'bursaria' groups of Paramecium (Miwa 1979). The molecular incompatibility such as the species specificity of immaturity substances may be involved in the inter- 
specific conjugation. Alternatively, cytoplasmic connection may not occur. The presence of meiosis-inducing factor (MIF) in Paramecium is suggested by micronuclear transplantation (Fujishima 1981) and the MIF is known to be effective in Xenopus oocyte (Fujishima, personal communication). Nuclear changes would not synchronize without cytoplasmic exchange even if conjugation-regulating molecules such as MIF are commonly effective beyond the barrier of species.

Autogamy occurs naturally in $P$. tetraurelia or can be induced artificially in P. multimicronucleatum and P. caudatum (Miyake 1968; Tsukii and Hiwatashi 1979; Mikami and Koizumi 1979). Thus, cell union is not indispensable for nuclear activation in the 'aurelia' group. However, no induction of autogamy has been reported in P. bursaria.

In order to attempt artificial induction of autogamy in P. bursaria, two kinds of experiments were performed. Single mating reactive cells were placed into depression slides separately with conjugation-inducing chemicals. Under this condition, each cell can not adhere. Ten hrs after transfer into chemical agents, cells were fixed and stained, and whether cells went through meiosis or not was examined. Although more than a thousand cells were observed, nuclear activation did not occur. It is known that when protease is added to the conjugation-inducing chemicals at various times from the start of the induction of conjugation, conjugants once united are gradually disjoined, and the separated cells undergo autogamy in $P$. caudatum (Tsukii and Hiwatashi 1979). Similar experiments were performed in $P$. bursaria as the second attempt of induction of autogamy. Although trypsin, pronase $\mathrm{P}$ and papain were used at various concentrations, no nuclear activation was detected. These results suggest that nuclear activation in $P$. bursaria is induced only when cell adhesion occurs even though its interaction is interspecific. In relation to this point, conjugation of Tetrahymena differs from that of Paramecium. Though homotypic pairs consisting of the same mating type are transiently formed in Tetrahymena, nuclear activation does not occur (Kitamura et al. 1986). Mating type specific interactions may be involved in the trigger mechanism of nuclear activation in Tetrahymena but not in that of Paramecium. Cell adhesion after mating reaction and nuclear activation of Paramecium occur in non-species-specific manner.

The author thanks Ms. Miranda Mirfakhrai and Dr. Yasuhiro Suzuki, my colleagues, for reading and correction of this manuscript. I also thank Dr. Toshiro Sugai, Ibaraki University, and Dr. Yuuji Tsukii, Hosei University, for critical reading and invaluable comments.

\section{REFERENCES}

Endoh, H., Watanabe, T. and Hiwatashi, K. (1987) Artificial induction of selfing conjugation in Paramecium bursaria. J. Exp. Zool. (in press).

Fujishima, M. and Hiwatashi, K. (1981) Transplantation of germ nuclei in Paramecium cauda- 
tum. II. Induction of meiosis in transplanted interphase nucleus. Exp. Cell Res. 131, 6371.

Hiwatashi, K. (1951) Studies on the conjugation of Paramecium caudatum IV. Conjugation between individuals of two mating types marked by a vital staining method. Sci. Rep. Tohoku Univ. (Biol). 19, 95-99.

Hiwatashi, K. (1968) Determination and inheritance of mating type in Paramecium caudatum. Genetics 58, 373-386.

Hiwatashi, K. (1981) Sexual interactions of the cell surface in Paramecium. In: Sexual interactions in Eukaryotic Microbes. (eds. O'Day, D. H. and Horgan, P. A.) pp. 351-378. Academic Press, New York.

Hiwatashi, K. (1985) Fertilization of Paramecium. In: Biology of fertilization. (eds. Metz, C. B. and Monroy, A.) Vol. 1, pp. 57-85. Academic Press, New York.

Kitamura, A., Sugai, T. and Kitamura, Y. (1986) Homotypic pair formation during conjugation in Tetrahymena thermophila. J. Cell Sci. 82, 223-234.

Metz, C. B. (1954) Mating substances and the physiology of fertilization in ciliates. In: Sex in Microorganisms. (eds. Whenrich, D. H.). pp. 284-334. Am. Assoc. Adv. Sci., Washington, D. C.

Mikami, K. and Korzumi, S. (1979) Induction of autogamy by treatment with trypsin in Paramecium caudatum. J. Cell Sci. 35, 177-184.

Miwa, I. (1979) Specificity of the immaturity substances in Paramecium. J. Cell Sci. 36, 253260.

MiYak E, A. (1958) Induction of conjugation by chemical agents in Paramecium caudatum. $J$. Inst. Polytech. Osaka City Univ. (Biol.) 9, 251-296.

Miyake, A. (1968) Induction of conjugation by chemical agents in Paramecium. J. Exp. Zool. 167, 359-380.

Myohara, K. and Hiwatashi, K. (1978) Mutants of sexual maturity in Paramecium caudatum selected by erythromycin resistance. Genetics 90, 227-241.

Sonneborn, T. M. (1937) Sex, sex inheritance and sex determination in Paramecium aurelia. Proc. Natl. Acad. Sci. U.S. 23, 378-385.

SonneboRn, T. M. (1957) Breeding systems, reproductive methods, and species problem in Protozoa. In: The Species Problem. (eds. Mayer, E.) pp. 155-324. Am. Assoc. Advan. Sci. Washington, D. C.

Sonneborn, T. M. (1974) Paramecium aurelia. In: Handbook of Genetics, vol. 2. pp. 469-594. (eds. King, R. C.) Plenum Press, New York.

Sugai, T. and Hiwatashi, K. (1974) Cytologic and autoradiographic studies of the micronucleus at meiotic prophase in Tetrahymena pyriformis. J. Protozool. 21, 542-548.

SugaI, T. (1976) Chromosomal association and arrangement at meiotic prophase of the micronucleus in Paramecium. Jpn. J. Genetics 51, 385-392.

TsukiI, Y. and Hiwatashi, K. (1979) Artificial induction of autogamy in Paramecium caudatum. Genet. Res. 34, 163-172.

TsukiI, Y. and Hiwatashi, K. (1983) Genes controlling mating-type specificity in Paramecium caudatum: Three loci revealed by intersyngenic crosses. Genetics 104, 41-62.

Usuki, I. and IRIE, T. (1983a) Inter- and intrasyngenic variation of Paramecium hemoglobin. I. Paramecium aurelia complex. Comp. Biochem. Physiol. 75B, 415-420.

UsUKI, I. and IRIE, T. (1983b) Inter- and intrasyngenic variation of Paramecium hemoglobinII. Paramecium caudatum, Paramecium jenningsi and Paramecium multimicronuclea. tum. Comp. Biochem. Physiol. 75B, 421-424.

WATANABE, T. (1978) A scanning electron-microscopic study of the local degeneration of cilia during sexual reproduction in Paramecium. J. Cell Sci. 32, 55-66.

Wichterman, R. (1986) Biology of Paramecium. 2nd eds. Plenum Press, New York. 